\title{
Many-level fuzzy rough approximation spaces induced by many-level fuzzy preorders and the related ditopological structures
}

\author{
Alexander Šostak ${ }^{a, b}$ and Alexandrs Eljkins ${ }^{b}$ and Ingrīda Uljane ${ }^{a, b}$ \\ ${ }^{a}$ Institute of Mathematics and CS University of Latvia, \\ aleksandrs.sostaks@lumii.lv, Ingrida Uljane@lumii.lv \\ ${ }^{b}$ University of Latvia, Department of Mathematics, \\ aleksandrs.sostaks@lu.lv, aleksandrs.elkins@gmail.com, ingrida.uljane@lu.lv
}

\begin{abstract}
We present a many-level version for the Pawlak - Dubois\&Prade theory of rough approximation of fuzzy sets. Basing on the many-level upper and lower fuzzy rough approximation operators, we define the measure of rough approximation that in a certain sense characterizes the quality of the obtained approximation. Further, the fuzzy rough approximation operators give rise to two alternative topological-type structures considered in the paper.
\end{abstract}

Keywords: Many-level fuzzy rough approximation operators, measure of fuzzy rough approximation, $L M$-fuzzy (di)topologies, $M$ level $L$-fuzzy (di)-topologies.

\section{Introduction}

A problem that became especially actual in the last quarter of the previous century was to deal with information systems using big volumes of data and other similar situations. Answering this challenge, Z. Pawlak in his celebrated paper [11] has introduced the concept of a rough set and developed the basics of the corresponding theory. In [4] D. Dubois and H. Prade have introduced a fuzzy version of a rough set; later the theory of fuzzy rough sets was developed in different directions. In this paper, we present a many-level version of rough approximation for $L$-fuzzy sets and develop a method that allows to estimate, in a certain sense, the quality of this approximation. Further, we present here a certain motivation for many-level approach in the theory of rough approximation of fuzzy sets.

One of possible interpretations of a rough set is as follows. Assume we are looking from some distance at a plane filled up with pixels, and $D$ is a domain in this plane. Then we may be sure that a pixel, say $p$, is in the domain $D$. Let $l(D)$ be the set of all such pixels. Further, for some pixels, we may be hesitating whether they are inside $D$ or not. Let $b d(D)$ be the set of all such pixels and let $u(D)=l(D) \cup b d(D)$. Obviously, $l(D)$ and $u(D)$ can be viewed as respectively the lower and the upper Pawlak's rough approximations of the domain $D$ and $b d(D)$ as its boundary set. But now imagine that we change the distance from which the observation is made. Then we can expect that the lower and upper rough approximations of the domain $D$ vary depending on the distance from which the observation is made. Thus lower and upper rough approximations of $D$ become functions $l_{d}(D)$ and $u_{d}(D)$ of the parameter $d$ (the distance of observation). To manage with this and other similar cases in crisp, as well as in fuzzy cases, one can use many-level lower and upper fuzzy rough approximation operators. Further, we develop a method allowing to estimate the quality of obtained approximation. It is based on the measures of approximation introduced here. Continuing the previous example, these measures characterize, respectively, "how precisely $D$ is covered by $u_{d}(D)$ " and "how precisely $l_{d}(D)$ is covered by $D$ ".

Another example. Assume we make an approximation of an object. It may happen that at some stages this approximation is not as precise as at the others. And the transfer from lq'less precise" to "more precise" should be done in a "smooth" way. Our approach presents a model how this transition can be done.

\section{Many-level $L$-fuzzy relations}

\subsection{Basic definitions}

Let $L=\left(L, \leq_{L}, \wedge_{L}, \vee_{L}, *\right)$ be an integral commutative complete lattice monoid (in particular, $L=[0,1]$ and * a lower semi-continuous $t$-norm), see, e.g. [8], and let $M=\left(M, \leq_{M}, \wedge_{M}, \vee_{M}\right)$ be a complete infinitely distributive lattice.

Definition 2.1 An M-level L-fuzzy relation on a set 
$X$ is a mapping $R: X \times X \times M \rightarrow L$. An M-level $L$-fuzzy relation on a set $X$ is called:

1. reflexive, if $R(x, x, \alpha)=1_{L} \forall x \in X, \alpha \in M$;

2. separated, if $R(x, y, \alpha)=1_{L} \forall \alpha \in M \Longrightarrow x=y$;

3. symmetric, if $R(x, y, \alpha)=R(y, x, \alpha) \forall x, y \in$ $X, \alpha \in M$;

4. transitive, if $R(x, y, \alpha) * R(y, z, \alpha) \leq$ $R(x, z, \alpha) \forall x, y, z \in X, \alpha \in M$.

5. A reflexive transitive $M$-level $L$-fuzzy relation is called an $M$-level $L$-fuzzy preoder or an $L M$ fuzzy preoder for short.

A pair $(X, R)$, where $X$ is a set and $R: X \times X \times$ $M \rightarrow L$ is an $L M$-fuzzy preoder, is called an $L M$ fuzzy preodered space.

Above we considered level-wise properties of an $M$ level $L$-fuzzy relation $R$. Now we collect properties showing the behavior of the relation $R$ between different levels $\alpha \in M$.

Definition 2.2 An $M$-level L-fuzzy relation $R$ on a set $X$ is called

1. non-increasing, if $\alpha \leq \beta \Longrightarrow R(x, y, \alpha) \geq$ $R(x, y, \beta) \forall x, y \in X, \forall \alpha, \beta \in M$;

2. upper semi-continuous, if $R\left(x, y, \bigvee_{i \in I} \alpha_{i}\right)=$ $\bigwedge_{i \in I} R\left(x, y, \alpha_{i}\right) \forall x, y \in X, \forall\left\{\alpha_{i} \mid i \in I\right\} \subseteq M$.

3. lower semi-continuous, if $R\left(x, y, \bigwedge_{i \in I} \alpha_{i}\right)=$ $\bigvee_{i \in I} R\left(x, y, \alpha_{i}\right) \forall x, y \in X, \forall\left\{\alpha_{i} \mid i \in I\right\} \subseteq M$;

4. global, if it satisfies conditions $(\perp)$ and $(\top)$ :

$(\perp) R\left(x, y, 0_{M}\right)=1_{L}$ for all $x, y \in X$;

( $\top$ ) $R\left(x, y, 1_{M}\right)=1_{L}$ if $x=y$ and

$R\left(x, y, 1_{M}\right)=0_{L}$ otherwise .

Given $L M$-fuzzy preodered spaces $\left(X, R_{X}\right)$ and $\left(Y, R_{Y}\right)$, a mapping $f: X \rightarrow Y$ is called monotone non-decreasing, or just monotone for short if $R_{X}\left(x, x^{\prime}, \alpha\right) \leq R_{Y}\left(f(x), f\left(x^{\prime}\right), \alpha\right)$ for all $x, x^{\prime} \in X$ and all $\alpha \in M$.

The category of $L M$-fuzzy preodered spaces and their monotone mappings is denoted by $L M$-PREL.

\subsection{Construction of an $M$-level $L$-fuzzy relation from a quasi-pseudometric}

We construct an $M$-level $L$-fuzzy relation $R_{\rho}$ from an ordinary quasi-pseudometric $\rho$ on a set $X$. A similar construction in case $\rho$ is a metric was considered in
[16] where it was used in the study of many-valued bornologies.

Let $L=M=[0,1]$ be the unit intervals viewed as lattices and let $*: L \times L \rightarrow L$ be a continuous $t$-norm. Further, let $X$ be a set and $\rho: X \times X \rightarrow[0,1]$ be a quasi-pseudometric on this set. We define an $M$-level $L$-fuzzy relation $R_{\rho}: X \times X \times[0,1] \rightarrow[0,1]$ by setting

$R_{\rho}(x, y, \alpha)=\left\{\begin{aligned} \frac{\alpha}{\alpha+(1-\alpha) \rho(x, y)} & \text { if } \alpha \neq 0 \text { or } \rho(x, y) \neq 0 \\ 1 & \text { otherwise. }\end{aligned}\right.$

It is easy to see that $R_{\rho}(x, y, \cdot):[0,1] \rightarrow[0,1]$ is continuous for all $x, y \in[0,1]$. Modifying the proof of Proposition 7.1 in [16] we can get the following result:

Theorem 2.3 For any quasi-pseudometric $\rho: X \times$ $X \rightarrow[0,1]$, many-level fuzzy relation $R_{\rho}: X \times X \times$ $[0,1] \rightarrow[0,1]$ is reflexive, upper and lower semicontinuous and global. If $\rho$ is a pseudometric, then $R_{\rho}$ is symmetric. If $\rho$ is a quasi-metric, then $R_{\rho}$ is separated. The relation $R_{\rho}$ is transitive in cases of the product $t$-norm $*=$. and hence for any weaker continuous t-norm. If $\rho$ is an ultra pseudometric, then relation $R_{\rho}$ is transitive for any continuous t-norm *.

Corollary 2.4 In cases $*=$. and $*=*_{L}$ the mapping $R_{\rho}: X \times X \times[0,1] \rightarrow[0,1]$ is a global continuous LM-fuzzy preoder for any quasi-pseudpmetric $\rho: X \times X \rightarrow[0,1]$. If $\rho$ is an ultra pseudometric, then $R_{\rho}$ is a global continuous LM-fuzzy preoder for any continuous t-norm.

Remark 2.5 If we start with an arbitrary quasipseudometric $d: X \times X \rightarrow[0, \infty)$, then for the definition of the relation $R_{d}$ we can take its equivalent quasi-pseudometric $\rho: X \times X \rightarrow[0,1]$ defined by $\rho(x, y)=\frac{d(x, y)}{1+d(x, y)}$. In this case $M$-level $L$-fuzzy relation $R_{\rho}$ can be rewritten as $R_{d}(x, y, \alpha)=\frac{\alpha(1+d(x, y))}{\alpha+d(x, y)}$ if $\alpha \neq 0$ or $d(x, y) \neq 0$ and $R_{d}(x, y, 0)=1$ if $d(x, y)=0$.

\section{$3 \quad M$-level rough approximation of an $L$-fuzzy set}

Given an $L M$-fuzzy preoder $R: X \times X \times M \rightarrow L$, we define the upper $M$-level $L$-rough approximation operator $u_{R}: L^{X} \times M \rightarrow L^{X}$ and the lower $M$-level $L$ rough approximation operator $l_{R}: L^{X} \times M \rightarrow L^{X}$ by $u_{R}(A, \alpha)(x)=\bigvee_{y}\left(R(y, x, \alpha) * A(y)\right.$ and $l_{R}(A, \alpha)(x)=$ $\bigwedge_{y}\left(R(x, y, \alpha) \mapsto A(y) \quad \forall A \in L^{X}\right.$, respectively.

Theorem 3.1 $M$-level L-rough approximation operators satisfy the following properties:

(1u) $u_{R}\left(a_{X}, \alpha\right)=a_{X} \forall \alpha \in M$ where $a_{X}: X \rightarrow L$ is the constant function with value a;

(2u) $A \leq u_{R}(A, \alpha) \forall A \in L^{X}, \forall \alpha \in M$; 
(3u) $u_{R}\left(\bigvee_{i} A_{i}, \alpha\right)=\bigvee_{i} u_{R}\left(A_{i}, \alpha\right)$

$\forall\left\{A_{i} \mid i \in I\right\} \subseteq L^{X} \forall \alpha \in M$;

(4u) $u_{R}\left(u_{R}(A, \alpha), \alpha\right)=u_{R}(A, \alpha) \forall A \in L^{X} \forall \alpha \in M$;

(5u) $\alpha \leq \beta \Longrightarrow u_{R}(A, \alpha) \geq u_{R}(A, \beta)$

(11) $l_{R}\left(a_{X}, \alpha\right)=a_{X} \forall \alpha \in M$;

(21) $A \geq l_{R}(A, \alpha) \forall A \in L^{X} \forall \alpha \in M$;

(3l) $l_{R}\left(\bigwedge_{i} A_{i}, \alpha\right)=\bigwedge_{i} l_{R}\left(A_{i}, \alpha\right)$

$\forall\left\{A_{i} \mid i \in I\right\} \subseteq L^{X} \forall \alpha \in M$;

(4l) $l_{R}\left(l_{R}(A, \alpha), \alpha\right)=l_{R}(A, \alpha) \forall A \in L^{X} \forall \alpha \in M$.

(5l) $\alpha \leq \beta \Longrightarrow l_{R}(A, \alpha) \leq l_{R}(A, \beta)$

Besides, if $R$ is lower semi-continuous, then

(6u) $u_{R}\left(A, \bigwedge_{j} \alpha_{j}\right)=\bigvee_{j}\left(u_{R}\left(A, \alpha_{j}\right) \forall\left\{\alpha_{j} \mid j \in J\right\} \subseteq A\right.$;

(61) $l_{R}\left(A, \bigwedge_{j} \alpha_{j}\right)=\bigwedge_{j}\left(l_{R}\left(A, \alpha_{j}\right) \forall\left\{\alpha_{j} \mid j \in J\right\} \subseteq A\right.$

Proof We omit the proofs of properties $(1 u)-(4 u)$ and (11) - (4l) since they can be easily obtained by modifying the proofs of the corresponding properties of fuzzy rough approximation operators in the proof of Proposition 4 in [12] for the many-level case. Properties $(5 \mathrm{u})$ and $(5 \mathrm{l})$ are clear from the definitions. To prove properties (6u) and (6l) let $A \in L^{X}, x \in X$ and $\left\{\alpha_{j}, \mid j \in J\right\} \subset M$ be given. Then

$$
\begin{gathered}
u_{R}\left(A, \bigwedge_{j} a_{j}\right)(x)=\bigvee_{y} R\left(y, x, \bigwedge_{j} \alpha_{j}\right) * A(y)= \\
\bigvee_{y}\left(\bigvee_{j} R\left(y, x, \alpha_{j}\right) * A(y)\right)= \\
\bigvee_{j} \bigvee_{y}\left(R\left(y, x, \alpha_{j}\right) * A(y)\right)=\bigvee_{j}\left(u_{R}\left(A, \alpha_{j}\right)(x)\right.
\end{gathered}
$$

and

$$
\begin{gathered}
l_{R}\left(A, \bigwedge_{j} \alpha_{j}\right)(x)=\bigwedge_{y}\left(R\left(x, y, \bigwedge_{j} \alpha_{j}\right) \mapsto A(y)\right)= \\
\bigwedge_{y}\left(\bigvee_{j} R\left(x, y, \alpha_{j}\right) \mapsto A(y)\right)= \\
\bigwedge_{y} \bigwedge_{j}\left(R\left(x, y, \alpha_{j}\right) \mapsto A(y)\right)= \\
\bigwedge_{j}\left(\bigwedge_{y} R\left(x, y, \alpha_{j}\right) \mapsto A(y)\right)=\bigwedge_{j} l_{R}\left(A, \alpha_{j}\right)(x) .
\end{gathered}
$$

The proof of the following theorem can be done by level-wise modification in the proofs of Proposition 3 and Theorem 1 in [12].

Theorem 3.2 The equalities $u_{R}\left(l_{R}(A, \alpha), \alpha\right)=$ $l_{R}(A, \alpha)$ and $l_{R}\left(u_{R}(A, \alpha), \alpha\right)=u_{R}(A, \alpha)$ hold for every $L$-fuzzy set $A$ and every $\alpha \in M$,.

Definition 3.3 The pair $\left(u_{R}, l_{R}\right)$, where $R: X \times X \times$ $M \rightarrow L$ is an LM-fuzzy preoder on $X$ and $u_{R}, l_{R}$ : $L^{X} \times M \rightarrow L^{X}$ are $M$-level $L$-rough approximation operators is called an $M$-level $L$-rough approximative pair, and the corresponding triple $\left(X, u_{R}, l_{R}\right)$ is called an $M$-level $L$-rough approximation space.

Definition 3.4 Let $\left(X, u_{R_{X}}, l_{R_{X}}\right)$ and $\left(Y, u_{R_{Y}}, l_{R_{Y}}\right)$ be $M$-level $L$-rough approximation spaces. A mapping $f: X \rightarrow Y$ is called continuous ${ }^{1}$ if it satisfies the following two conditions:

(con 1) $f\left(u_{R_{X}}(A, \alpha)\right) \leq u_{R_{Y}}(f(A), \alpha) \forall A \in L^{X} \forall \alpha \in$ $M$,

(con 2) $f^{-1}\left(l_{R_{Y}}(B, \beta)\right) \leq l_{R_{X}}\left(f^{-1}(B, \beta)\right) \quad \forall B \quad \in$ $L^{Y}, \forall \beta \in M$.

By $L M$-RAS we denote the category of $M$-level $L$ rough approximation spaces and their continuous mappings.

Theorem 3.5 If $f:\left(X, R_{X}\right) \rightarrow\left(Y, R_{Y}\right)$ is a monotone mapping then the mapping $f:\left(X, u_{R_{X}}, l_{R_{X}}\right) \rightarrow$ $\left(Y, u_{R_{Y}}, l_{R_{Y}}\right)$ is continuous.

Proof To prove the first property, we fix $A \in L^{X}$, $\alpha \in M, y \in Y$ and reason as follows:

$$
\begin{gathered}
f\left(u_{R_{X}}(A, \alpha)\right)(y)=\bigvee_{f(x)=y} u_{R_{X}}(A, \alpha)(x)= \\
\bigvee_{f(x)=y}\left(\bigvee_{x^{\prime}} R\left(x^{\prime}, x, \alpha\right) * A\left(x^{\prime}\right)\right) \leq \\
\bigvee_{x^{\prime}} R_{Y}\left(f\left(x^{\prime}\right), f(x), \alpha\right) * A\left(x^{\prime}\right)= \\
\bigvee_{x^{\prime}} R_{Y}\left(f\left(x^{\prime}\right), y, \alpha\right) * A\left(x^{\prime}\right) \leq \\
\bigvee_{y^{\prime}} R_{Y}\left(y^{\prime}, y, \alpha\right) * f(A)\left(y^{\prime}\right)=u_{R_{Y}}(f(A), \alpha)(y) .
\end{gathered}
$$

To prove the second property, we fix $B \in L^{Y}, \beta \in M$, $x \in X$ and reason as follows:

$$
\begin{gathered}
f^{-1}\left(l_{R_{Y}}(B, \beta)(x)\right)=l_{R_{Y}}(B, \alpha) f(x)= \\
\bigwedge_{y^{\prime}} R_{Y}\left(f(x), y^{\prime}, \beta\right) \mapsto B\left(y^{\prime}\right) \leq \\
\bigwedge_{x^{\prime}} R_{Y}\left(f(x), f\left(x^{\prime}\right)\right)(\beta) \mapsto B\left(f\left(x^{\prime}\right)\right) \leq \\
\bigwedge_{x^{\prime}} R_{X}\left(x, x^{\prime}, \beta\right) \mapsto B\left(f\left(x^{\prime}\right)\right)= \\
\bigwedge_{x^{\prime}} R_{X}\left(x, x^{\prime}, \beta\right) \mapsto f^{-1}(B)\left(x^{\prime}\right)=l_{R_{X}}\left(f^{-1}(B, \beta)\right)(x) .
\end{gathered}
$$

Corollary 3.6 Assigning the space $\left(X, u_{R_{X}}, l_{R_{X}}\right)$ to an LM-fuzzy preodered set $(X, R)$ and interpreting a monotone mapping $f:\left(X, R_{X}\right) \rightarrow\left(Y, R_{Y}\right)$ as a mapping $f:\left(X, u_{R_{X}}, l_{R_{X}}\right) \rightarrow\left(Y, u_{R_{Y}}, l_{R_{Y}}\right)$, we get an embedding functor from the category LM-PREL of LMfuzzy preodered sets into the category LM-RAS.

\footnotetext{
${ }^{1}$ the justification of this term will be clarified later
} 


\section{$4 \quad L M$-fuzzy ditopology induced by rough approximation operators on an $L M$-fuzzy preodered set}

Many-level upper and lower $L$-rough approximation operators give rise to two ditopological type structures on the underlying set. These ditopologies will be the subject of this and the next section. However, first we have to specify terminology concerning topological structures in fuzzy environment. Following the terminology initiated in [9] and now accepted by many authors, by an $L$-topology on a set $X$ we call a family $T \subseteq L^{X}$ of its $L$-fuzzy subsets such that $0_{L} \in T ; U, V \in T \Rightarrow U \wedge V \in T$ and $U_{i} \in T \forall i \in$ $I \Rightarrow \bigvee_{i} U_{i} \in T$. On the other hand, by an $L M$-fuzzy topology we call a mapping $\mathcal{T}: L^{X} \rightarrow M$ such that $\mathcal{T}\left(0_{X}\right)=1_{M} ; U, V \in L^{X} \Rightarrow \mathcal{T}(U \wedge V) \geq \mathcal{T}(U) \wedge \mathcal{T}(V) ;$ $\mathcal{T}\left(\bigvee_{i} U_{i}\right) \geq \bigwedge_{i} \mathcal{T}\left(U_{i}\right)$. In an analogous way, an $L$-cotopology and an $L M$-fuzzy co-topology on a set $X$ are defined. In case $L=M$ we just speak about $L$ topologies and $L$-co-topologies. Since in the context of our research $L$-topologies ( $L M$-fuzzy topologies) and $L$-co-topologies (resp. $L M$-fuzzy co-topologies) generally are unrelated, we stick to the terminology introduced in the works by M. L. Brown and his co-authors, see e.g. [2], and use the terms $L$-ditopology and $L M$ fuzzy ditopology respectively.

\section{1 $L M$-fuzzy topology on an $L M$-preordered set}

Let $(X, R)$ be an $L M$-fuzzy preordered set and let $l_{R}: L^{X} \times M \rightarrow L^{X}$ be the lower $L M$-rough approximation operator induced on this set. Then the properties $(1 l)-(4 l)$ of $l_{R}$ collected in Theorem 3.1 mean that for every level $\alpha$ the restriction of the mapping $l_{R}$ to the set $L^{X} \times\{\alpha\}$, that is the mapping $l_{R}: L^{X} \times\{\alpha\} \rightarrow L^{X}$ can be interpreted as the interior operator on $L^{X}$ see, e.g. [10], [13], [14]). In particular, if $R$ is global, then $l_{R}\left(A, 0_{M}\right)(x)=\inf _{y \in X} A(y)$ and $l_{R}\left(A, 1_{M}\right)(x)=A(x)$

Hence, by setting $T_{\alpha}=\left\{A \in L^{X}: l_{R}(A, \alpha)=A\right\}$, we obtain the $L$-topology corresponding to this $L$ fuzzy interior operator. Moreover, the property $(3 l)$ allows to conclude that it is actually an Alexandroff $L$-topology (see e.g. [1], [3]), that is the intersection axiom holds also for infinite families of $L$-fuzzy sets. Thus for each $\alpha$ the family $T_{\alpha}$ satisfies the axioms of an Alexandroff $L$-topology:

1. $0_{X}, 1_{X} \in T_{\alpha}$;

2. $\left\{A_{i}: i \in I\right\} \subseteq T_{\alpha} \Longrightarrow \bigwedge_{i} A_{i} \in T_{\alpha}$;

3. $\left\{A_{i}: i \in I\right\} \subseteq T_{\alpha} \Longrightarrow \bigvee_{i} A_{i} \in T_{\alpha}$

Taking such $L$-topologies for all $\alpha \in M$, we obtain the family $\left\{T_{\alpha}: \alpha \in M\right\}$. Besides, since $l_{R}(A, \beta) \leq$
$l_{R}(A, \alpha)$ whenever $\beta \leq \alpha$, we conclude that

$$
\beta \leq \alpha \Longrightarrow T_{\beta} \subseteq T_{\alpha},
$$

that is the family $\left\{T_{\alpha}: \alpha \in M\right\}$ is non-decreasing. In particular, $T_{0}=\left\{a_{X} \mid a: X \rightarrow L^{X}\right\}$. This means that $T_{0}$ consists of all constants and hence is the indiscrete stratified $L$-topology. On the other hand $T_{1}=L^{X}$, that is $T_{1}$ is the discrete $L$-topology.

We use this family in order to construct an $L M$-fuzzy topology from this indexed set of $L$-topologies. To do this in a coordinated way, in addition, we assume that $M$ is a De Morgan algebras, that is a completely distributive lattice endowed with an order reversing involution ${ }^{c}: M \rightarrow M$. We define

$$
\mathcal{T}(A)=\bigvee\left\{\alpha \in M: A \in T_{\alpha^{c}}\right\}
$$

Theorem 4.1 If the lattice $M$ is completely distributive, then $\mathcal{T}$ is an LM-fuzzy topology on the LMpreordered set $(X, R)$, that is

$$
\begin{aligned}
& \text { 1. } \mathcal{T}\left(0_{X}\right)=0_{M} \\
& \text { 2. } \mathcal{T}\left(\bigwedge_{i} A_{i}\right) \geq \bigwedge_{i} \mathcal{T}\left(A_{i}\right) \text { for all }\left\{A_{i}: i \in I\right\} \subseteq L^{X} \\
& \text { 3. } \mathcal{T}\left(\bigvee_{i} A_{i}\right) \geq \bigwedge_{i} \mathcal{T}\left(A_{i}\right) \text { for all }\left\{A_{i}: i \in I\right\} \subseteq L^{X}
\end{aligned}
$$

Proof The first property is obvious, since $0_{X} \in T_{\alpha}$ for all $\alpha \in M$.

To prove the second property, take any family $\left\{A_{i}\right.$ : $i \in I\} \subseteq L^{X}$ and assume that $\bigwedge_{i} \mathcal{T}\left(A_{i}\right)=\alpha$. In case $\alpha=0_{M}$ the inequality is obvious, therefore we assume that $\alpha>0_{M}$. Take any $\beta \triangleleft \alpha$ where $\triangleleft$ is the wedge below relation on the completely distributive lattice $M$. From the definition of $\mathcal{T}$ it is clear that $A_{i} \in T_{\beta^{c}}$ for every $i \in I$ and hence, recalling that $T_{\beta^{c}}$ is an Alexandroff $L$-topology, we conclude that also $\bigwedge_{i} A_{i} \in$ $T_{\beta^{c}}$. Therefore $\mathcal{T}\left(\bigwedge_{i} A_{i}\right) \geq \beta^{c}$. Since this is true for any $\beta \triangleleft \alpha$ and lattice $M$ is completely distributive, we conclude that $\mathcal{T}\left(\bigwedge_{i} A_{i}\right) \geq \alpha^{c}=\bigwedge_{i} \mathcal{T}\left(A_{i}\right)$.

To prove the third property, take any family $\left\{A_{i}\right.$ : $i \in I\} \subseteq L^{X}$ and assume that $\bigvee_{i} \mathcal{T}\left(A_{i}\right)=\alpha$. In case $\alpha=0_{M}$ the inequality is obvious, therefore we assume that $\alpha>0_{M}$. Take any $\beta \triangleleft \alpha$. From the definition of $\mathcal{T}$ it is clear that $A_{i} \in T_{\beta^{c}}$ for every $i \in$ $I$ and hence, recalling that $T_{\beta^{c}}$ is an $L$-topology, we conclude that also $\bigvee_{i} A_{i} \in T_{\beta^{c}}$. Therefore $\mathcal{T}\left(\bigwedge_{i} A_{i}\right) \geq$ $\beta$. Since this is true for any $\beta \triangleleft \alpha$ and the lattice $M$ is completely distributive, we conclude that $\mathcal{T}\left(\bigvee_{i} A_{i}\right) \geq$ $\alpha=\bigwedge_{i} \mathcal{T}\left(A_{i}\right)$.

\section{2 $L M$-fuzzy co-topology on an $L M$-preorderd set}

Let $(X, R)$ be an $L M$-fuzzy preordered set and let $u_{R}: L^{X} \times M \rightarrow L^{X}$ be the upper $L$-rough approximation operator induced by the $L M$-relation $R$ on 
the set $X$. Then properties $(1 u)-(4 u)$ of the upper $L M$-rough approximation operator $u_{R}$ mean that the restriction of $u_{R}$ to $L^{X} \times\{\alpha\}$, can be interpreted as an $L$-fuzzy closure operator on the set $L^{X}$ (This fact is well-known, see, e.g. [10], [13], [14]). Besides,

$$
\alpha \leq \beta \Longrightarrow u_{R}(A, \alpha) \geq u_{R}(A, \beta) ;
$$

$u_{R}\left(A, 0_{M}\right)(x)=\sup _{y \in X} A(y), \quad u_{R}\left(A, 1_{M}\right)(x)=A(x)$.

Now, given $\alpha \in M$ the family $K_{\alpha}=\left\{A \in L^{X}\right.$ : $\left.u_{R}(A, \alpha)=A\right\}$, is the Alexandroff $L$-co-topology corresponding to this $L$-fuzzy closure operator. This means that

1. $1_{X} \in K_{\alpha}$;

2. $\left\{A_{i}: i \in I\right\} \subseteq K_{\alpha} \Longrightarrow \bigvee_{i} A_{i} \in K_{\alpha}$;

3. $\left\{A_{i}: i \in I\right\} \subseteq K_{\alpha} \Longrightarrow \bigwedge_{i} A_{i} \in K_{\alpha}$

Taking such $L$ - co-topologies for all $\alpha \in M$, we obtain the family $\left\{K_{\alpha}: \alpha \in M\right\}$. Besides, since

$$
\beta \leq \alpha \Longrightarrow u_{R}(\cdot, \beta) \geq u_{R}(\cdot, \alpha)
$$

we conclude that

$$
\beta \leq \alpha \Longrightarrow K_{\beta} \subset K_{\alpha},
$$

that is the family $\left\{K_{\alpha}: \alpha \in M\right\}$ is non-decreasing. To use this family of $L$-co-topologies in order to define an (Alexandroff) $L M$-fuzzy co-topology $\mathcal{K}$ on the set $X$, as in the previous subsection, we assume that lattice $M$ is completely distributive and is endowed with an order reversing involution ${ }^{c}: M \rightarrow M$. Now, by setting

$$
\mathcal{K}(A)=\bigvee\left\{\alpha \in M: A \in K_{\alpha^{c}}\right\}
$$

we obtain a mapping $\mathcal{K}: L^{X} \times M \rightarrow L^{X}$.

Theorem 4.2 $\mathcal{K}$ is an LM-fuzzy co-topology on the set $X$, that is

$$
\begin{aligned}
& \text { 1. } \mathcal{K}\left(1_{X}\right)=1_{M} ; \\
& \text { 2. } \mathcal{K}\left(\bigvee_{i} A_{i}\right) \geq \bigwedge_{i} \mathcal{K}\left(A_{i}\right) \text { for all }\left\{A_{i}: i \in I\right\} \subseteq L^{X} ; \\
& \text { 3. } \mathcal{K}\left(\bigwedge_{i} A_{i}\right) \geq \bigwedge_{i} \mathcal{K}\left(A_{i}\right) \text { for all }\left\{A_{i}: i \in I\right\} \subseteq L^{X}
\end{aligned}
$$

Proof The first property is obvious, since $1_{X} \in K_{\alpha}$ for all $\alpha \in M$.

To prove the second property, take any family $\left\{A_{i}\right.$ : $i \in I\} \subseteq L^{X}$ and assume that $\bigwedge_{i} \mathcal{K}\left(A_{i}\right)=\alpha$. In case $\alpha=0_{M}$ the inequality is obvious, therefore we assume that $\alpha>0_{M}$. Take any $\beta \triangleleft \alpha$ where $\triangleleft$ is the wedge-below relation in the completely distributive lattice $M$. Then from the definition of $\mathcal{K}$ it is clear that $A_{i} \in K_{\beta^{c}}$ for every $i \in I$, and hence, recalling that $K_{\beta^{c}}$ is an Alexandroff $L$-co-topology, we conclude that also $\bigvee_{i} A_{i} \in K_{\beta^{c}}$. Therefore $\mathcal{K}\left(\bigvee_{i} A_{i}\right) \geq \beta$. Since this is true for any $\beta \triangleleft \alpha$ and lattice $M$ is completely distributive, we conclude that $\mathcal{K}\left(\bigvee_{i} A_{i}\right) \geq \alpha=\bigwedge_{i} \mathcal{K}\left(A_{i}\right)$.

To prove the third property, take any family $\left\{A_{i}: i \in\right.$ $I\} \subseteq L^{X}$ and assume that $\bigvee_{i} \mathcal{K}\left(A_{i}\right)=\alpha$. In case $\alpha=0_{M}$ the inequality is obvious, therefore we assume that $\alpha>0_{M}$. Take any $\beta \triangleleft \alpha$. Then from the definition of $\mathcal{K}$ it is clear that $A_{i} \in K_{\beta^{c}}$ for every $i \in I$, and hence, recalling that $K_{\beta^{c}}$ is an Alexandroff $L$-cotopology, we conclude that also $\bigvee_{i} A_{i} \in K_{\beta}^{c}$. Therefore $\mathcal{K}\left(\bigvee_{i} A_{i}\right) \geq \beta$. Since this is true for any $\beta \triangleleft \alpha$ and lattice $M$ is completely distributive, we conclude that $\mathcal{K}\left(\bigwedge_{i} A_{i}\right) \geq \alpha=\bigwedge_{i} \mathcal{K}\left(A_{i}\right)$.

\subsection{The case of an $M V$-algebra}

In this section we assume that $L$ is an $M V$-algebra, see e.g. [6]. This means that $\left(\alpha \mapsto 0_{L}\right) \mapsto 0_{L}=\alpha$, and hence by setting $\alpha^{\prime}=\alpha \mapsto 0_{L}$, we obtain an order reversing involution' $: L \rightarrow L$ on the lattice $L$. This involution is extended point-wise to the $L$-powerset $L^{X}$ as $A^{\prime}(x)=A(x) \mapsto 0_{L}$. Besides, we continue to assume that $M$ is a De Morgan algebra.

Generally $L M$-fuzzy topology $\mathcal{T}$ and $L M$-fuzzy cotopology constructed above are unrelated and hence we cannot view the obtained structures as an $L M$ fuzzy topology. As we will see here, in case $L$ is an $M V$-algebra and the $L M$-fuzzy preorder relation $R$ on a set $X$ is symmetric, then the pair $(\mathcal{T}, \mathcal{K})$ is an $L$-fuzzy topology where the mapping $\mathcal{T}: L^{X} \rightarrow M$ determines the degree of openness of $L$-fuzzy subsets of $X$ while the mapping $\mathcal{K}: L^{X} \rightarrow M$ determines the degree of closeness of $L$-fuzzy subsets of $X$ and $\mathcal{T}\left(A^{\prime}, \alpha\right)=\mathcal{K}(\alpha, A)^{\prime}$ for every $A \in L^{X}$.

Indeed, it is easy to notice that in case of an $M V$ algebra $L$ and the symmetric $L$-fuzzy preoder $R$, we have $u_{R}(A, \alpha)^{\prime}=l_{R}\left(A^{\prime}, \alpha\right)$ for every $A \in L^{X}$ and every $\alpha \in M$.

Recalling the definition of $L$-topology $T_{\alpha}$ and $L$-cotopology $K_{\alpha}$, for every $A \in L^{X}$ we have:

$$
\begin{aligned}
A \in K_{\alpha} & \Longleftrightarrow u_{R}(A, \alpha)=A \Longleftrightarrow \\
u_{R}(A, \alpha)^{\prime}=A^{\prime} & \Longleftrightarrow l_{R}\left(A^{\prime}, \alpha\right)=A^{\prime} \Longleftrightarrow A \in T_{\alpha} .
\end{aligned}
$$

Now we have

$$
\begin{gathered}
\mathcal{K}(A)=\bigvee\left\{\alpha \in M \mid A \in K_{\alpha^{c}}\right\}= \\
\bigvee\left\{\alpha \in M \mid u_{R}\left(A, \alpha^{c}\right)=A\right\}= \\
\bigvee\left\{\alpha \in M \mid u_{R}\left(A, \alpha^{c}\right)^{\prime}=A^{\prime}\right\}= \\
\bigvee\left\{\alpha \in M \mid l_{R}\left(A^{\prime}, \alpha^{c}\right)=A^{\prime}\right\}=\mathcal{T}\left(A^{\prime}\right) .
\end{gathered}
$$


Thus we have the following theorem:

Theorem 4.3 If $L$ is an $M V$-algebra and the $L M$ fuzzy relation $R$ on a set $X$, then $\mathcal{T}(A)=\mathcal{K}\left(A^{\prime}\right)$ for every $A \in L^{X}$ and hence the mapping $\mathcal{T}: L^{X} \rightarrow M$ is an $L M$-fuzzy topology on $X$.

\section{$5 \quad$ Measure of $M$-level $L$-fuzzy rough approximation and induced $M$-level $L$-fuzzy ditopologies}

\subsection{Measure of inclusion of $L$-fuzzy sets}

Definition 5.1 By setting $A \hookrightarrow B=\bigwedge_{x \in X}(A(x) \mapsto$ $B(x))$ where $A, B \in L^{X}$ and $\mapsto: L \times L \rightarrow L$ is the residuum corresponding to the operation $*$ by Galois connection, we obtain a mapping $\hookrightarrow: L^{X} \times L^{X} \rightarrow L$. We call $A \hookrightarrow B$ by the measure of inclusion of the $L$-fuzzy set $A$ into the L-fuzzy set $B$.

Proposition 5.2 Relation $\hookrightarrow: L^{X} \times L^{X} \rightarrow L$ satisfies the following properties for all $\left\{A_{i} \mid i \in I\right\} \subseteq L^{X}$, $A \in L^{X},\left\{B_{i} \mid i \in I\right\} \subseteq L^{X}, B \in L^{Y}$ :

(1) $\left(\bigvee_{i} A_{i}\right) \hookrightarrow B=\bigwedge_{i}\left(A_{i} \hookrightarrow B\right)$;

(2) $A \hookrightarrow\left(\bigwedge_{i} B_{i}\right)=\bigwedge_{i}\left(A \hookrightarrow B_{i}\right)$;

(3) $A \hookrightarrow B=1_{L}$ whenever $A \leq B$;

(4) $1_{X} \hookrightarrow A=\bigwedge_{x} A(x)$;

(5) $(A \hookrightarrow B) \leq(A * C \hookrightarrow B * C) \forall A, B, C \in L^{X}$;

(6) $(A \hookrightarrow B) *(B \hookrightarrow C) \leq(A \hookrightarrow C) \forall A, B, C \in L^{X}$;

(7) $\left(\bigwedge_{i} A_{i}\right) \hookrightarrow\left(\bigwedge_{i} B_{i}\right) \geq \bigwedge_{i}\left(A_{i} \hookrightarrow B_{i}\right)$;

(8) $\left(\bigvee_{i} A_{i}\right) \hookrightarrow\left(\bigvee_{i} B_{i}\right) \geq \bigwedge_{i}\left(A_{i} \hookrightarrow B_{i}\right)$

The proof of these properties is easy and can be found in the recent works of different authors.

\subsection{Measure of $M$-level $L$-fuzzy rough approximation}

Let $(X, R)$ be an $L M$-fuzzy preodered set. Given an $L$-fuzzy set $A \in L^{X}$, we define the measure $\mathcal{U}(A, \cdot)$ : $M \rightarrow L$ of its upper $M$-level $L$-fuzzy rough approximation by $\mathcal{U}_{R}(A, \alpha)=u_{R}(A, \alpha) \hookrightarrow A$. Respectively, the measure $\mathcal{L}(A, \cdot): M \rightarrow L$ of its lower $M$-level $L$-fuzzy rough approximation is defined by $\mathcal{L}_{R}(A, \alpha)=A \hookrightarrow l_{R}(A, \alpha)$. If $R$ is symmetric then it is easy to see that $\mathcal{U}_{R}(A, \alpha)=\mathcal{L}_{R}(A, \alpha)$ for every $L$-fuzzy set $A$. In this case we call it by the measure of $M$-level $L$-fuzzy rough approximation of an $L$-fuzzy set $A$.
The above defined measures of lower and upper $M$ level rough approximation of $L$-fuzzy sets give rise to the $M$-level operators of upper and lower $L$-fuzzy rough approximation $\mathcal{U}_{R}: L^{X} \times M \rightarrow L$ and $\mathcal{L}_{R}$ : $L^{X} \times M \rightarrow L$ and the operator of $M$-level $L$-fuzzy rough approximation $\mathfrak{R}_{R}: L^{X} \times M \rightarrow L$ in case $R$ is symmetric. In the next theorem we collect the main properties of these operators.

Theorem 5.3 1. $\mathcal{U}_{R}\left(a_{X}, \alpha\right)=1_{L} \forall \alpha \in M ;$

2. $\mathcal{L}_{R}\left(a_{X}, \alpha\right)=1_{L} \forall \alpha \in M$;

3. $\mathcal{U}_{R}\left(u_{R}(A, \alpha), \alpha\right)=1_{L} \forall A \in L^{X}, \forall \alpha \in M$;

4. $\left.\mathcal{L}_{R}\left(l_{R}(A), \alpha\right), \alpha\right)=1_{L} \forall A \in L^{X} \forall \alpha \in M$;

5. $\mathcal{U}_{R}\left(\bigvee_{i} A_{i}, \alpha\right) \geq \bigwedge_{i} \mathcal{U}_{R}\left(A_{i}, \alpha\right)$ $\forall\left\{A_{i} \mid i \in I\right\} \subseteq L^{X} \forall \alpha \in M$;

6. $\mathcal{U}_{R}\left(\bigwedge_{i} A_{i}, \alpha\right) \geq \bigwedge_{i} \mathcal{U}_{R}\left(A_{i}, \alpha\right)$ $\forall\left\{A_{i} \mid i \in I\right\} \subseteq L^{X}, \forall \alpha \in M$;

7. $\mathcal{L}_{R}\left(\bigwedge_{i} A_{i}, \alpha\right) \geq \bigwedge_{i} \mathcal{L}_{R}\left(A_{i}, \alpha\right)$ $\forall\left\{A_{i} \mid i \in I\right\} \subseteq L^{X}, \forall \alpha \in M$;

8. $\mathcal{L}_{R}\left(\bigvee_{i} A_{i}, \alpha\right) \geq \bigwedge_{i} \mathcal{L}_{R}\left(A_{i}, \alpha\right)$ $\forall\left\{A_{i} \mid i \in I\right\} \subseteq L^{X}, \forall \alpha \in M$

9. $\mathcal{U}_{R}\left(a_{X} * A, \alpha\right) \geq \mathcal{U}_{R}(A, \alpha)$ for all $A \in L^{X}$ and all constants $a_{X}$

10. $\mathcal{L}_{R}\left(a_{X} \mapsto A, \alpha\right) \geq \mathcal{L}_{R}(A, \alpha)$ for all $A \in L^{X}$ and all constants $a_{X}$.

Proof (1) Referring to Theorem 3.1 and applying Proposition 5.2 we have $\mathcal{U}_{R}\left(a_{X}, \alpha\right)=u_{R}\left(a_{X}, \alpha\right) \hookrightarrow$ $a_{X}=a_{X} \hookrightarrow a_{X}=1_{M}$.

(2) Referring to Theorem 3.1 and applying Proposition 5.2 , we have

$\mathcal{L}_{R}\left(a_{X}, \alpha\right)=a_{X} \hookrightarrow l_{R}\left(a_{X}, \alpha\right)=a_{X} \hookrightarrow a_{X}=1_{M}$.

(3) Referring to Theorem 3.1 and applying Proposition 5.2 , we have $\mathcal{U}_{R}\left(u_{R}(A, \alpha)\right)=u_{R}\left(u_{R}(A, \alpha), \alpha\right) \hookrightarrow$ $u_{R}(A, \alpha)=u_{R}(A, \alpha) \hookrightarrow u_{R}(A, \alpha)=1_{M}$.

(4) Referring to Theorem 3.1 and applying Proposition 5.2 , we have $\mathcal{L}_{R}\left(l_{R}(A, \alpha), \alpha\right)=l_{R}(A, \alpha) \hookrightarrow$ $l_{R}\left(l_{R}(A, \alpha), \alpha\right)=l_{R}(A, \alpha) \hookrightarrow l_{R}(A, \alpha)=1_{M}$

(5) Referring to Theorem 3.1 and applying Proposition 5.2 , we have $\mathcal{U}_{R}\left(\bigvee_{i} A_{i}, \alpha\right)=u_{R}\left(\bigvee_{i} A_{i}, \alpha\right) \hookrightarrow \bigvee_{i} A_{i}=$ $\bigvee_{i} u_{R}\left(A_{i}, \alpha\right) \hookrightarrow \bigvee_{i} A_{i}$

(6) Referring to Theorem 3.1 and applying Proposition 5.2 we have $\mathcal{U}_{R}\left(\bigwedge_{i} A_{i}, \alpha\right)=u_{R}\left(\bigwedge_{i} A_{i}, \alpha\right) \hookrightarrow \bigwedge_{i} A_{i} \geq$ $\bigwedge_{i} u_{R}(A, \alpha) \hookrightarrow \bigwedge_{i} A_{i} \geq \bigwedge_{i}\left(u_{R}\left(A_{i}, \alpha\right) \hookrightarrow A_{i}\right)=$ $\bigwedge_{i} \mathcal{U}_{R}\left(A_{i}, \alpha\right)$.

(7) Referring to Theorem 3.1 and applying Proposition 5.2, we have $\mathcal{L}_{R}\left(\bigwedge_{i} A_{i}, \alpha\right)=\left(\bigwedge_{i} A_{i}\right) \hookrightarrow l_{R}\left(\bigwedge_{i} A_{i}, \alpha\right)=$ $\bigwedge_{i} A_{i} \hookrightarrow \bigwedge_{i} l_{R}\left(A_{i}, \alpha\right) \geq \bigwedge_{i} A_{i} \hookrightarrow l_{R}\left(\bigwedge_{i} A_{i}, \alpha\right)$.

(8) Referring to Theorem 3.1 and applying and applying Proposition 5.2, we have $\mathcal{L}_{R}\left(\bigvee_{i} A_{i}, \alpha\right)=$ $\left(\bigvee_{i} A_{i}\right) \hookrightarrow l_{R}\left(\bigvee_{i} A_{i}, \alpha\right) \geq \bigwedge_{i} A_{i} \hookrightarrow \bigwedge_{i} l_{R}\left(A_{i}, \alpha\right) \geq$ 
$\bigvee_{i} A_{i} \hookrightarrow l_{R}\left(\bigvee_{i} A_{i}, \alpha\right) \geq \bigwedge_{i}\left(l_{R}\left(A_{i}, \alpha\right) \hookrightarrow A_{i}\right)=$ $\bigwedge_{i} \mathcal{L}\left(A_{i}, \alpha\right)$.

(9) $\mathcal{U}_{R}(\alpha * A, \alpha)=u(\alpha * A, \alpha) \hookrightarrow \alpha *$ $A)=\bigwedge_{x \in X} \bigvee_{x^{\prime} \in X}\left(R\left(x^{\prime}, x, \alpha\right) * A\left(x^{\prime}\right) * \alpha \mapsto \alpha *\right.$ $A(x)) \geq \bigwedge_{x \in X} \bigvee_{x^{\prime}}\left(R\left(x^{\prime}, x, \alpha\right) * A\left(x^{\prime}\right) \mapsto A(x)\right)=$ $\bigwedge_{x \in X}\left(u_{R}(A, \alpha)\left(x^{\prime}\right) \mapsto A(x)\right)=\mathcal{U}_{R}(A, \alpha) \hookrightarrow A ;$

(10) Recall first that for every $x$ we have $\alpha \mapsto$ $l_{R}(A, \alpha)(x)=l_{R}(A \mapsto \alpha)(x)$. Indeed, $\alpha \mapsto$ $l_{R}(A, \alpha)(x)=\alpha \mapsto \bigwedge_{x^{\prime}}\left(R\left(x^{\prime}, x, \alpha\right) \mapsto A\left(x^{\prime}\right)\right)=$ $\bigwedge_{x^{\prime}}\left(\alpha \mapsto\left(R\left(x^{\prime}, x, \alpha\right) \mapsto A\left(x^{\prime}\right)\right)=\bigwedge_{x^{\prime}}\left(R\left(x^{\prime}, x, \alpha\right) \mapsto\right.\right.$ $\left(\alpha \mapsto A\left(x^{\prime}\right)\right)=l_{R}(\alpha \mapsto A, \alpha)(x)$.

Now we get the requested inequality as follows:

$\mathcal{L}_{R}(A, \alpha)=A \hookrightarrow l_{R}(A, \alpha)=\bigwedge_{x}(A(x) \mapsto$ $\left.l_{R}(A, \alpha)(x)\right) \leq \bigwedge_{x}(\alpha \mapsto A)(x) \mapsto\left(\alpha \mapsto l_{R}(A, \alpha)(x)=\right.$ $\bigwedge_{x}(\alpha \mapsto A)(x) \mapsto\left(\alpha \mapsto l_{R}(A, \alpha)(x)\right)=$ $\mathcal{L}_{R}(\alpha \mapsto A, \alpha)$.

\subsection{Examples of measures for many-level fuzzy rough approximation of $L$-fuzzy sets}

The case of Łukasiewicz $t$-norm Let $*_{L}$ be the Lukasiewicz t-norm on the interval $L=[0,1]$, and $\mapsto_{L}$ : $L \times L \rightarrow L$ be the corresponding residuum. Then, given an $M$-level $L$-fuzzy relation $R$ on a set $X, A \in$ $L^{X}$ and $\alpha \in M$, we have:

$\mathcal{U}_{R}(A, \alpha)=\bigwedge_{x} \bigwedge_{x^{\prime}}\left(2-A(x)+A\left(x^{\prime}\right)-R\left(x, x^{\prime}, \alpha\right)\right)$

$\mathcal{L}_{R}(A, \alpha)=\bigwedge_{x} \bigwedge_{x^{\prime}}\left(2-A(x)+A\left(x^{\prime}\right)-R\left(x^{\prime}, x, \alpha\right)\right)$.

In particular, if $R: X \times X \times M \rightarrow[0,1]$ is global, then $\mathcal{R}_{R}\left(A, 0_{M}\right)=1_{L}$ and $\mathcal{R}_{R}\left(A, 1_{M}\right)=1-\inf _{x, x^{\prime}} \mid$ $A(x)-A\left(x^{\prime}\right) \mid$ for all $A \in L^{X}$.

The case of the minimum $t$-norm Let $*=\wedge$ be the minimum $t$-norm on the unit interval $L=[0,1]$, and $\mapsto: L \times L$ be the corresponding residuum, Then $\mathcal{U}_{R}(A, \alpha)=\inf _{x, x^{\prime}}\left(A\left(x^{\prime}\right) \wedge R\left(x, x^{\prime}, \alpha\right) \mapsto A(x)\right)$, $\mathcal{L}_{R}(A, \alpha)=\inf _{x, x^{\prime}}\left(A\left(x^{\prime}\right) \wedge R\left(x^{\prime}, x, \alpha\right) \mapsto A(x)\right)$. In particular, if $R$ is global, then $\mathcal{R}_{R}(A, \alpha)=1_{L} \forall A \in$ $L^{X}, \alpha \in M$.

The case of the product $t$-norm Let $*=$ be the product $t$-norm on the unit interval $[0,1]$ and $\mapsto: L \times L$ be the corresponding residuum, Then

$\mathcal{U}(A, \alpha)=\inf _{x, x^{\prime}}\left(A\left(x^{\prime}\right) \cdot R\left(x, x^{\prime}, \alpha\right) \mapsto A(x)\right)$,

$\mathcal{L}(A, \alpha)=\inf _{x, x^{\prime}}\left(A\left(x^{\prime}\right) \cdot R\left(x^{\prime}, x, \alpha\right) \mapsto A(x)\right)$. In particular in case $R$ is global, $\mathcal{R}_{R}\left(A, 1_{M}\right)=1_{L}$ and $\mathcal{R}_{R}\left(A, 0_{M}\right)=\inf _{x, x^{\prime} \in X} \frac{A(x)}{A\left(x^{\prime}\right)}$.

\subsection{On the category of $M$-level $L$-fuzzy rough approximation spaces}

Given an $L M$-fuzzy preodered set $(X, R)$, the quadruple $\left(X, R, \mathcal{U}_{R}, \mathcal{L}_{R}\right)$ is called by an $M$-level $L$-fuzzy rough approximation space.

Definition 5.4 We call a mapping of $M$-level L-fuzzy rough approximation spaces $f:\left(X, \mathcal{U}_{R_{X}}, \mathcal{L}_{R_{X}}\right) \rightarrow$
$\left(Y, \mathcal{U}_{R_{Y}}, \mathcal{L}_{R_{Y}}\right)$ continuous if

(1con) $\mathcal{U}_{R_{X}}\left(f^{-1}(B), \alpha\right) \geq \mathcal{U}_{Y}(B, \alpha) \forall B \in L^{Y}, \forall \alpha \in$ $M$;

(2con) $\mathcal{L}_{R_{X}}\left(f^{-1}(B), \alpha\right) \geq \mathcal{L}_{Y}(B, \alpha) \forall B \in L^{Y}$.

Let $M L$-FRAS be the category whose objects are $M$ level L-fuzzy rough approximation spaces and whose morphisms are continuous mappings.

By straightforward verification one can easily prove the following statement:

Theorem 5.5 Let $R_{X}: X \times X \times M \rightarrow L$ and $R_{Y}: Y \times Y \times M \rightarrow L$ be $L M$-fuzzy preoders on sets $X$ and $Y$ respectively and let $f:\left(X, R_{X}\right) \rightarrow$ $\left(Y, R_{Y}\right)$ be a monotone mapping. Then the mapping $f:\left(X, \mathcal{U}_{R_{X}}, \mathcal{L}_{R_{X}}\right) \rightarrow\left(Y, \mathcal{U}_{R_{Y}}, \mathcal{L}_{R_{Y}}\right)$ is continuous.

Thus assigning the $M$-level $L$-fuzzy rough approximation space $\left(X, \mathcal{U}_{R_{X}}, \mathcal{L}_{R_{X}}\right)$ to an $M$-level $L$-fuzzy preoder space $(X, R)$ and interpreting monotone mappings $f:\left(X, R_{X}\right) \rightarrow\left(Y, R_{Y}\right)$ as mappings $f$ : $\left(X, \mathcal{U}_{R_{X}}, \mathcal{L}_{R_{X}}\right) \rightarrow\left(Y, \mathcal{U}_{R_{Y}}, \mathcal{L}_{R_{Y}}\right)$, we obtain an embedding functor from the category $L M$-PREL of $L M$ fuzzy preodered sets into the category $L M$-RAS.

\subsection{Ditopological interpretation of $M$-level $L$-fuzzy rough approximation spaces}

Let $\left(X, \mathcal{L}_{R}, \mathcal{U}_{R}\right)$ be an $M$-level $L$-fuzzy $L$-rough approximation space and let $\alpha \in M$ be fixed. Properties (1), (5) and (6) of Theorem 5.3 characterize the relation's $\mathcal{L}_{R}: L^{X} \times M \rightarrow L$ restriction to the set $L^{X} \times\{\alpha\}$ as a stratified $L$-fuzzy topology on the set $X$ [10], [9]. In its turn, properties (2), (7) and (8) characterize the relation's $\mathcal{U}_{R}: L^{X} \times M \rightarrow L$ restriction to the set $L^{X} \times\{\alpha\}$ as a stratified $L$-fuzzy co-topology on a set $X$. This observation justifies the following definition:

Definition 5.6 An M-level L-fuzzy topology on a set $X$ is a mapping $\mathcal{L}_{R}: L^{X} \times M \rightarrow L$ satisfying properties (1), (5) and (6) of Theorem 5.3. Respectively, an $M$ level $L$-fuzzy co-topology on a set $X$ is a mapping $\mathcal{U}_{R}$ : $L^{X} \times M \rightarrow L$ satisfying properties (2), (7) and (8) of Theorem 5.3.

Now from Theorem 5.5 we get the following:

Theorem 5.7 Let $(X, R)$ be an $L M$-fuzzy preodered set. Then the triple $\left(X, \mathcal{L}_{X}, \mathcal{U}_{X}\right)$ is a stratified $M$-level $L$-fuzzy ditopology.

Theorem 5.8 By assigning the $M$-level L-fuzzy ditopological space $\left(X, \mathcal{L}_{R_{X}}, \mathcal{U}_{R_{X}}\right)$ to an LM-fuzzypreodered set $(X, R)$ and interpreting monotone mappings $f:\left(X, R_{X}\right) \rightarrow\left(Y, R_{Y}\right)$ as mappings of the corresponding $M$-level $L$-fuzzy ditopological spaces $f$ : 
$\left(X, \mathcal{L}_{R_{X}}, \mathcal{U}_{R_{X}}\right) \rightarrow\left(Y, \mathcal{L}_{R_{Y}}, \mathcal{U}_{R_{Y}}\right)$, we get an embedding functor from the category of $L M$-fuzzy preordered sets into the category of $M$-level stratified $L$-fuzzy ditopological spaces.

\section{Conclusions}

Basing on the research done in our papers [5], [15], [16], [7], we initiate here the many level approach to rough approximation for L-fuzzy sets, introduce the measure of the quality of this approximation and illustrate it with examples. Our special attention in this paper is made to two alternative topological interpretations of this approximation. As the main perspectives for the further work, we see both developing theoretical aspects of many-level rough fuzzy approximation of fuzzy sets, and applications to problems of practical nature. Concerning the theoretical issues, as first, we plan to develop further the qualitative approach to the theory of many-level fuzzy rough approximation for $L$-fuzzy sets in the framework of category theory. An investigation of the relations between the manylevel approach to rough approximation and the theory of multigranual rough sets [17] is also one of the prospectives for the future work. As one of possible applications of our approach to practical problems, we see image processing. The idea of this application was sketched by an example in the Introduction. Besides, we guess that our approach could be helpful when studying some problems of decision making in fuzzy environment.

\section{Acknowledgement}

The first and the last named authors are thankful for the partial financial support from the project No Lzp2018/2-0338 'Development of fuzzy logic based technologies for risk assessment by means of relationalgrounded aggregation' by the Latvian Council of Science.

All authors are thankfull to the anonymous referees for reading the paper carefully and making some critical comments.

\section{References}

[1] Alexandroff, P: Diskrete Räume. Mat. Sbornik 2 (1937), 501-518.

[2] Brown, L.M., Ertürk, R., Dost Ş. : Ditopological texture spaces and fuzzy topology, I. Basic concepts. Fuzzy Sets and Syst. 110 (2000), 227-236.

[3] Chen, P., Zhang, D. : Alexandroff $L$-cotopological spaces. Fuzzy Sets and Systems 161 (2010) 2505 -2525 .
[4] Dubois, D., Prade, H.: Rough fuzzy sets and fuzzy rough sets. Intern. J. General Systems 17 (1990), 191-209.

[5] Ellkins, A., Šostak, A., Uljjane, I.: On a category of extensional fuzzy rough approximation operators. In: Communication in Computer Inform. Sci., vol 611 (2016).

[6] Hájek, P.: Methamatimatics of Fuzzy Logic, Kluwer Acad. Publ., 1998.

[7] Han, S-E., Šostak, A.: On the measure of Mrough approximation of L-fuzzy sets. Soft Computing 22 (2018), 2843-2855.

[8] Höhle, U.: M-valued sets and sheaves over integral commutative cl-monoids, Chapter 2 In: S.E. Rodabaugh, E.P. Klement, U. Höhle (eds.) Applications of Category Theory to Fuzzy Sets. Kluwer Acad. Publ. (1992), pp. 33-73.

[9] Höhle, U., Šostak, A.: Axiomatic foundations of fixed-based fuzzy topology, In: Höhle, U., Rodabaugh, S. (eds.) Mathematics of Fuzzy Sets: Logic, Topology and Measure Theory, pp. 123272 Kluwer Acad. Publ. (1999).

[10] Liu, Y-M., Luo, M-K.: Fuzzy Topology Advances in Fuzzy Systems - Applications and Topology. World Scientif. Singapore, New Jersey, London, Hong Kong, 1997.

[11] Pawlak, Z.: Rough sets. Intern. J. of Computer and Inform. Sci. 11 (1982), 341-356.

[12] Eļkins, A. Šostak, A., Uljjane, I.: On a category of extensional fuzzy rough approximation operators. In: Communication in Computer and Information Science, vol 611 (2016). 16. International Conference IPMU 2016, Eindhofen, The Netherlands, June 20-24, 2016, Proceedings, Part II, pp. $36-47$.

[13] Šostak, A.: Two decades of fuzzy topology: Basic ideas, notions and results, Russian Math. Surveys, 44 (1989), 125-186

[14] Šostak, A.: Basic structures of fuzzy topology, J. Math. Sci. 78 (1996), 662-701.

[15] Šostak, A., Elkins, A.: $L M$-valued equalities, $L M$-rough approximation operators and MLgraded ditopologies. Hacettepe J. Math. Stat. 46 (2017), 15-32.

[16] Šstak A., Uljane, I.: Bornological structures on many-valued sets. RadHAZU Matematičke Znanosti 21 (2017), 145-170.

[17] Yao Y., She Y.: Rough set models in multigranual spaces. Inform. Sci. 327 (2016), 40-56. 